\title{
Horacio H. Godoy \\ La Integración de \\ América Latina y el proceso \\ del poder mundial
}

\begin{abstract}
Horacio H. Godoy, argentino, director de la Escuela Latinoamerıcana de Ciencia Politica y Administración Pública de FLAcso, graduado en Leyes y en Sociologia en la Universidad de La Plata, LLM en la Universidad de Yale. Es autor de El Imperio de la Ley en Cuba, publicado por la Comisión Internacional de Juristas y dirigió el estudio $y$ la publicación del Informe sobre el Fondo Fiduciario de Progreso Social del BD en los años I 963 y 64 . Ha publicado más de diez trabajos sobre aspectos politicos e institucionales de la integración.
\end{abstract}

\section{Introducción}

I. Los factores condicionantes de carácler mundial:

1. Cambio acelerado y unificación mundial.

2. La çoncentración del poder mundial.

3. La proletarización de naciones.

4. La actualización de las ideologias.

5. La escala mundial.

6. La participación, signö del futuro inmediato.

\section{La in tegración de América Latina:}

1. El proceso de integración latinoamericana dentro del proceso mundial del poder.

2. Los organismos internacionales.

3. El mercado común latinoamericano.

4. La dimensión continental.

III. La dimensión nacional de la integración latinoamericana:

1. Integración para el desarrollo y la independencia.

2. Algunas criticas a la integración.

3. La integración: proceso irreversible y tarea generacional. 
Para comprender mejor los alcances del proceso de integración de América Latina resulta conveniente utilizar tres niveles de análisis diferentes. Un nivel de alcance mundial que permita determinar, por lo menos en sus lineas generales, las caracteristicas fundamentales del cambio profundo y acelerado que se vive en el mundo y las tendencias que se adviertan en cuanto a la futura estructuración del poder mundial. Esta dimensión mundial acentúa los aspectos de la creciente interdependencia para analizarla en forma global, tomando al mundo entero como una unidad operativa. La dimensión mundial difiere, cualitativamente, del enfoque clásico internacional, en el que los estados independientes son la unidad operativa del sistema ${ }^{1}$. La segunda dimensión de análisis corresponde al plano continental latinoamericano en el que entran a jugar los elementos comunes de las repúblicas latinoamericanas en términos culturales, sociales, económicos $y$ politicos. Y, por último, el nivel de análisis nacional en el que se determinan los valores e intereses propios de cada pais $y$ la posibilidad de su defensa $y$. de su desarrollo; se define la necesidad y la medida de la articulación de estos intereses en el plano continental latinoamericano; se establece la mejor manera de proyectar hacia el mundo los objetivos fundamentales de cada pais; y finalmente, se determinen las bases necesarias para lograr una participación efectiva en las decisiones fundamentales que se adopten para el futuro orden mundial.

\section{LOS FACTORES CONDICIONANTES DE CARÁCTER MLNDIAL}

\section{Cambio acelerado y unificación mundial}

Parece necesario repetir con insistencia que la humanidad entera se encuentra viviendo un proceso de cambio acelerado que afecta las bases mismas de la convivencia humana en el mundo. La aceleración, el alcance ! la profundidad del cambio tienen expresiones muy concretas que es necesario determinar $y$ evaluar para lograr una conciencia clara de la responsabilidad que como ciudadanos de un pais, como miembros de un continente $y$, finalmente, como simples individuos tenemos en nuestros dias.

Constituyen un lugar común las continuas referencias que en todas partes del mundo se escuchan acerca de la nueva era que la humanidad empieza a $v_{i v i r^{2}}$. Todos conocemos el desarrollo alcanzado por la ciencia y por la

\footnotetext{
${ }^{2}$ Ver.Johan Galtung: on The Future of international ststem. Pkio-publication $N^{*}{ }^{25}-2 \mathrm{n}$, International Peace Kesearch Institute. Oslo, 1 gits, pp. kJU8/3ing.

Ver además Mrres S. MeDougal and Associates: Studies in World Public Order. Yale Universitw Press. New Haven, $1 g^{\prime k o .}$ Especialmente The identification and appraisal of diverse Sistems of public order, pp. $3 / \mathrm{fl}$.

${ }^{2}$ Ver Gadium et Spes: Constitution Pastorale. L'Eglıse dans Le Monde de Ce Temps, pp. 1ry/81. Spes. Paris, Igit,. Expose Preliminaire, $x$ Conferencia de las Naciones Unidas sobre Aplicación de la Ciencia de la Tecnologia en beneficio de los pueblos en vias de desarrollo, celebrada en Ginebra en 1914 .
} 
tecnologia contemporáneas. Son numerosos los estudios realizados $;$ los que se están realizando para determinar los efectos que esta revolución cientifica $y$ tecnológica tiene y tendrá en la vida de los hombres y de las mujeres de todo el mundo, en la vida de los pueblos y en las relaciones internacionales. Baste referirnos a la Conferencia de las Naciones Unidas sobre Aplicación de la Ciencia y de la Tecnologia en beneficio de los pueblos en vias de desarrollo, celebrada en Ginebra en 1963 , en la que se analizó el efecto posible de la ciencia y de la tecnologia en materia de recursos naturales, recursos humanos, agricultura, desarrollo industrial, transporte, salud y' nutrición, problemas sociales del desarrollo y de la urbanización, planificación, comunicación, etc.

La Humanidad ha entrado ya en la llamada era espacial, o era atómica. La Tierra está rodeada por unos 300 satélites artificiales que han revolucionado è campo de las comunicaciones', la meteorologia e, inclusive, algunos aspectos fundamentales de la estrategia militar. Un satélite puede dar la vuelta al mundo en apenas go minutos y tanto puede ser utilizado con fines cientificos como puede estar equipado con instrumentos de espionaje. Desde otro punto de vista, la aplicación de los nuevos inventos al transporte ha permitido concebir la construcción de los aviones supersónicos que disminuy'en en tres y hasta en cinco veces el tiempo para recorrer distancias entre paises. En estudios realizados sobre el tamaño relativo del mundo en función del tiempo que se tarda en recorrer las distancias, se demuestra que el mundo résulta actualmente 25 veces más pequeño de lo que era proporcionalmente a comienzos del siglo ${ }^{4}$. Otro efecto concreto de esta revolución cientifica y tecnológica es el que se refiere a la disponibilidad y al uso de la energia atómica que pone a disposición de la humanidad recursos energéticos hasta ahora jamás concebidos. Por último el perfeccionamiento de las computadoras electrónicas y su aplicación en todos los campos del saber y del obrar humano, se enfrenta con nuevos aspectos que afectarán la convivencia humanas. Estos hechos y otros de alcance similar constituyen como la infraestructura del proceso de unificación del mundo.

\section{La concentración del poder mundial}

Paralelamente con este proceso acelerado y creciente de unificación mundial, la ciencia y la tecnologia aplicadas van produciendo una enorme concentración de recursos en los paises industrializados, mientras que los llamados paises en desarrollo que no participan plenamente en este proceso,

\footnotetext{
${ }^{3}$ Ver L Sesco: Communication in the space age. Paris, $19^{\text {ks. }}$

"Ver Stanford Research Institute: Póssible nonmilitars scientifie developments and their potential impact on foreign polics problems of the United States. United States Forcign Policv. Compilation of Studies. N"s. 1.8. Vol. t. United States. Government Printing Oflice. Washington, I gikl.

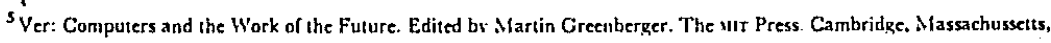
ignif.
} 
continuan su cvolución en el ritmo de otra época, lo que ha dado origen a la preocupación por el llamado vacio tecnológico.

El efecto de esta tambièn llamada segunda revolución industrial en la estructuración del poder mundial ha sido acrecentar la concentración del poder mundial en dos superpotencias colosales. Las superpotencias concentran el poder material en sus bases financieras, económicas, cientificas $y$ tecnológicas, que se traduce en una preeminencia absoluta en materia de comercio internacional; uso de la energia atómica; exploración y ocupación del espacio; ; utilización de las computadoras electrónicas gigantescas para la información y las comunicaciones.

Algunos ejemplos permitirán una mejor comprensión del alcance que tiene lo que llamamos el proceso de concentración del poder mundial:

a) El problema del oro y su relación con el dólar, lo resuelven los 7 paises que constituyen el Pool del oro, creado en Londres en 1961 . Estos paises son: Estados Unidos, Gran Bretaña, Alemania Occidental, Italia, Suiza, Bélgica y Holanda. Pero la reserva de oro de los Estados Unidos en marzo de $19 b 8$ era equivalente a 10,4 mil millones de dólares, más del doble de cualquier otro pais del mundo no socialista y casi 10 veces superior a la reserva total de los paises de América Latina. Los presidentes de los Bancos Centrales de estos siete paises estabiecieron un sistema doble del precio del oro para contener las compras especulativas que provocan la "fiebre del oro" manteniendo asi la relación del dólar con el oro.

b) El comercio internacional continúa beneficiando a los paises industrializados que representan apenas una tercera parte del total de paises y'menos de una tercera parte de la población mundial.

Se ha calculado que las exportaciones totales de los paises en vias de desarrollo o periféricos, excluidos los mayores exportadores de petróleo, crecerian en una tasa del $5 \%$, lo que provocaria un déficit potencial del intercambio (trade gap) hacia 1975 , del orden de los 24.000 millones de dólares; correspondiendo a América Látina un déficit equivalente a 8,2 mil millones de dólares ${ }^{6}$.

c) La disponibilidad de armas nucleares ha dividido a los paises del mundo en + categorias: 1) las superpotencias nucleares (Estados Unidos ! la Unión Soviética); 2) los poderes nucleares (Gran Bretaña, Francia y la República Popular China); 3) los llamados estados cercanos al poder nuclear (near nuclear powers), que por su desarrollo económico y capacidad tecnológica pueden llegar a fabricar armas nucleares (India, Japón, Canadá, Suiza, Republica Federal Alemana, Suecia, Israel y en América Latina probablemente Argentina ! Brasil), ! 4) finalmente los poderes no nucleares.

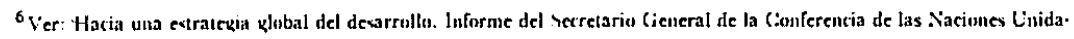

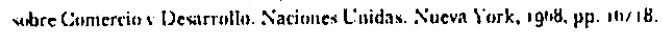

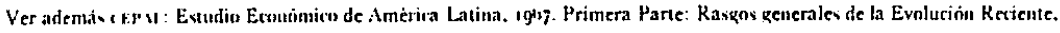
pp $2 \times 1$,
} 
El cuadro siguiente muestra la estructura del poder nuclear en el mundo:

\begin{tabular}{|c|c|c|c|}
\hline & & $\begin{array}{c}\text { Armas nucleares } \\
\text { (Bombas A y }{ }^{2} y \\
\text { cabezas nucleares) } \\
50.000 \text { on total }\end{array}$ & $\begin{array}{c}\text { Poder } \\
\text { (Megatones) }\end{array}$ \\
\hline $\begin{array}{l}\text { I Superpotencias } \\
\text { Nucleares }\end{array}$ & $\begin{array}{l}\text { EE.UU. } \\
\text { U. Soviética }\end{array}$ & $\begin{array}{l}30.000 \\
15.000\end{array}$ & $\begin{array}{l}25.000 \\
12.000 \\
\end{array}$ \\
\hline $\begin{array}{l}\text { II Poderes } \\
\text { Nucleares }\end{array}$ & $\begin{array}{l}\text { Gran Bretaña } \\
\text { Francia } \\
\text { China } \\
\end{array}$ & 5.000 & $\cdot$ \\
\hline $\begin{array}{l}\text { III Países cercanos } \\
\text { al poder } \\
\text { Nuclear }\end{array}$ & $\begin{array}{l}\text { India } \\
\text { Japón } \\
\text { Canadä, Suiza, } \\
\text { Suecia, Israel } \\
\text { (en América Latina, } \\
\text { Argentina y Brasil, } \\
\text { probablemente) }\end{array}$ & & . \\
\hline $\begin{array}{l}\text { IV Poderes no } \\
\text { Nucleares }\end{array}$ & $\begin{array}{l}120 \text { paises } \\
\text { independientes } \\
\text { aproximadamente }\end{array}$ & & \\
\hline
\end{tabular}

+ Un Megatón es igual a 50 bombas atónicas como la de Hiroshima e igual a 1 millón de tonelagdas de $\operatorname{TNT}^{7}$

La concentración del poder atómico en base a la disponibilidad de armas nucleares, en cantidades prácticamente inalcanzables por los demás paises, presenta el siguiente cuadro ${ }^{8}$.

Francia dispone de aviones de bombardeo del tipo Mirage iv con una capacidad de bo kilotones cada uno, que vuelan a dos veces la velocidad del sonido y pueden volar 4.000 kilómetros sin necesidad de abastecimiento. Para 1971 se prevé la disponibilidad de cohetes intercontinentales de tierra a tierra ( 9 cohetes) y de mar a tierra ( 16 cohetes). Los primeros tendrán una carga explosiva de 50 kilotones y alcanzarán a 2.500 kilómetros. Los segundos tendrán un alcance de 2.000 kilómetros y una carga explosiva de 500 kilotones. Está prevista también la construcción de otros 3 submarinos con 16 cohetes cada uno. Se ha señalado que en el caso más favorable, Francia dispondrá hacia 1975 de una capacidad nuclear calculada en 30 megatones, lo que equivale a la capacidad nuclear de un avión de bombardeo B-52

\footnotetext{
${ }^{7}$ Ver: Stafl Mernorandum: Current Status of The Anti-Ballistic Missile Program. Printed for the use of the Commiltce on Foreign Relations. U.S. Government Printing Orfice.

Washington: $19 n 7$. Ver también el interesante articulo de Alain. Joxe: Cohetes anticohetes: ;regreso a estratexias defensivas?

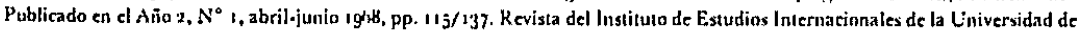
Chile. $v$ la conferencia de Adam Yarmolinskv: United States Military Power and Foreign Policv. The Universitr of Chicagn Center for Polics Studs. The Universitw of Chicago. 19:17, pp. 7/8.
}

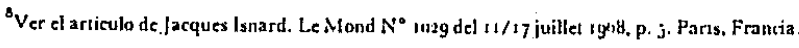




\begin{tabular}{|c|c|c|c|c|}
\hline Tipo & Pais & Unidades & $\begin{array}{c}\text { Potencia del } \\
\text { armamento p. Unidad }\end{array}$ & Alcance \\
\hline \multirow{5}{*}{$\begin{array}{l}\text { Bombar- } \\
\text { deros }\end{array}$} & U.S.A. & $\begin{array}{l}540 \text { subsónicos } \\
\text { pesados } \\
75 \begin{array}{l}\text { supersónicos } \\
\text { medios }\end{array}\end{array}$ & 10 a 25 megatones & \multirow{2}{*}{$\begin{array}{l}\text { Ilimitado con } \\
\text { reabasteci- } \\
\text { miento en } \\
\text { vuelo }\end{array}$} \\
\hline & U.R.S.S. & $\begin{array}{l}150 \text { subsónicos } \\
\text { pesados } \\
800 \text { supersónicos } \\
\text { medios }\end{array}$ & 1 megatón & \\
\hline & \multirow{2}{*}{$\begin{array}{l}\text { Gran } \\
\text { Bretaña }\end{array}$} & $\begin{array}{l}70 \text { subsónicos } \\
\text { Vy } \\
80 \text { Canberra }\end{array}$ & $\begin{array}{l}\text { Varios kilotones } \\
\text { a } 1 \text { megatón }\end{array}$ & \multirow{2}{*}{$\begin{array}{l}\text { Inferior a } \\
5.000 \\
\text { kilómetros }\end{array}$} \\
\hline & & $\begin{array}{l}60 \text { subsónicos } \\
\text { V en Alema- } \\
\text { nia del } \\
\text { Oeste } \\
\end{array}$ & $\begin{array}{l}\text { Cabezas nuclea- } \\
\text { res americanas }\end{array}$ & \\
\hline & China & \multicolumn{3}{|c|}{$\begin{array}{l}12 \text { Tupolov } 4 \text { y } 150 \text { Ilyouchine }-28 \text { subsónicos } \\
\text { A chorro: } 2.500 \text { kilómetros } \\
\text { Existencia evaluada en } 50 \text { bombas superiores a } \\
200 \text { kilotones }\end{array}$} \\
\hline \multirow{3}{*}{ Cohetes } & U.S.A. & $\begin{array}{c}1.100 \text { intercon- } \\
\text { tinentales }\end{array}$ & $\begin{array}{l}\text { De } 2 \text { a } 10 \text { megato- } \\
\text { nes }\end{array}$ & $\begin{array}{l}\text { De } 11.000 \text { a } \\
12.500 \text { kilómet }\end{array}$ \\
\hline & U.R.S.S. & $\begin{array}{c}700 \text { interconti- } \\
\text { nentales } \\
750 \text { distancia } \\
\text { media } \\
\text { Cohete orbital } \\
\text { Scrag } \\
\end{array}$ & $\begin{array}{l}\text { De } 10 \text { a } 50 \text { mega- } \\
\text { tones } \\
1 \text { megatón } \\
\text { De } 3 \text { a } 30 \text { megato- } \\
\text { nes }\end{array}$ & $\begin{array}{l}\text { De } 8.000 \text { a } \\
16.000 \text { kilómet } \\
\text { De } 1.700 \text { a } \\
3.400 \text { kilómets } \\
\text { Ilimitado }\end{array}$ \\
\hline & China & \multicolumn{3}{|c|}{$\begin{array}{l}\text { Un cohete experimentado en } 1966 \text { habría franqueado } \\
600 \text { kilómetros }\end{array}$} \\
\hline \multirow{4}{*}{$\begin{array}{l}\text { Submari- } \\
\text { nos estra- } \\
\text { tégicos }\end{array}$} & U.S.A & $\begin{array}{l}41 \text { equipados de } \\
16 \text { Polaris cada } \\
\text { uno }\end{array}$ & $\begin{array}{l}\text { De } 0,7 \text { a } 1 \text { mega } \\
\text { tón }\end{array}$ & $\begin{array}{l}\text { De } 2.800 \text { a } \\
4.500 \text { kilómets }\end{array}$ \\
\hline & U.R.S.S. & $\begin{array}{l}30 \text { a propulsión } \\
\text { nuclear - } 54 \text { a } \\
\text { propulsión tradi- } \\
\text { cional (c/u } 3 \text { a } \\
4 \text { cohetes balis- } \\
\text { ticos o aerodi- } \\
\text { námicos) }\end{array}$ & $\begin{array}{l}\text { De varios kiloto- } \\
\text { nes a } 1 \text { megatón }\end{array}$ & $\begin{array}{l}\text { De } 540 \text { a } \\
2.700 \text { kilómets. }\end{array}$ \\
\hline & Gran & $\begin{array}{l}1 \text { equipado de } \\
16 \text { Polaris }\end{array}$ & $\begin{array}{l}\text { Inferior a la ca- } \\
\text { beza americana }\end{array}$ & $\begin{array}{l}\text { Del orden de } \\
3.500 \mathrm{~km} \text {. }\end{array}$ \\
\hline & China & \multicolumn{3}{|c|}{$\begin{array}{l}1 \text { a } 3 \text { a propulsión tradicional, con } 3 \text { cohetes c/u. } \\
\text { con alcance de } 640 \text { kilómetros (préstamo soviético } \\
\text { de } 1964 \text { ) }\end{array}$} \\
\hline
\end{tabular}


norteamericano que puede transportar hasta 4 bombas $\mathrm{H}$ de 10 megatones cada una.

d) La exploración y conquista del espacio es prácticamente patrimonio de las dos superpotencias mundiales. Desde $195^{8}$ hasta el presente más de 300 satélites artificiales están girando sobre la tierra y se han lanzado casi un centenar de cohetes rusos y norteamericanos hacia los planetas Venus y Marte, hacia el Sol y hacia la Luna. Las estadisticas más recientes muestran que los Estados Unidos cuentan con 213 satélites artificiales en órbita, la Unión Soviética con 55, Francia con 3 y Canadá con 2. La utilización de estos satélites en las comunicaciones ${ }^{9}$, en estudios meteorológicos y su utilización como instrumentos de espionaje, define al espacio como un área estratégica de importancia decisiva para la estructuración del poder mundial. Las Naciones Unidas acaban de celebrar la primera conferencia sobre Exploración y Uso Pacifico del Espacio Exterior en Viena, a donde asistieron representantes de más de sesenta paises.

\section{La proletarización de naciones}

Dentro de este proceso global de unificación, el poder mundial sufre una doble transformación. Mientras los estados pequeños, débiles o subdesarrollados aumentan en cantidad y disminuyen en poder, las superpotencias aumentan en poder y disminuyen en cantidad. Este fenómeno se explica, por una parte, por lo que hemos llamado el proceso de "prolelarización de naciones" que coincide con la descolonización, $y$ consiguientemente, con la presencia de nuevos estados independiéntes; $y$, por otra parte, con la utilización de la ciencia y de la tecnologia moderna por las superpotencias, que aplicando estos conocimientos no sólo a la fabricación de nuevas armas nucleares, sino también a la conquista del espacio y a las nuevas industrias, acrecientan -en progresión geométrica- las diferencias de poder. Este doble proceso de dispersión y de concentración del poder mundial, tiene las caracteristicas de un proceso irreversible y la humanidad expresa a través de distintos medios y con voces diferentes sú preocupación por la búsqueda de los principios, de las instituciones y de los procedimientos para alcanzar un orden mundial en el que sobrevivan los valores. fundamentales de la persona humana ${ }^{10}$.

\section{La aclualización de las ideologias.}

Frente a este diagnóstico del poder mundial realizado en forma muy general; aparecen intentos de interpretación y comprensión humanista de la nueva era

\footnotetext{
${ }^{9}$ El Convenio Internacional de Salélites de Telecomunicaciones (INTELSaT) creado par acuerdo firmado en tgos, reune a más dr iw evtadus independientes, de los cuales 8 son latinoamericanos.

${ }^{10} \mathrm{Ver}$ The Strategy of World Order. Cuatro tomos. Editado por Richard A. Falk y Saul H. Mendlovitz. World Law Fund. Nini lork. (g)ks.
} 
en que vive la humanidad. $Y$ sin ambajes ni reservas se reconoce por la propia Iglesia Católica en la Constitución Conciliar que el sujeto de la historia contemporánea es la humanidad entera; que es la humanidad entera, nosotros, los pueblos del mundo - desde los más evolucionados hasta los más primitivos- los que somos reconocidos como los sujetos de la historia contemporánea.

Las ideologias de todos los sectores del mundo se esfuerzan por descubrir áreas de acción conjunta que ha dado lugar a que se piense en la posibilidad de un gran movimiento de convergencia para atender a las necesidades de los pueblos que sufren por el hambre y la miseria. Estas convergencias se producen en el plano del obrar concreto y resultan, por una parte, de la toma de conciencia de las necesidades que dos tercios de la humanidad padece, $y$ por la toma de conciencia de las posibilidades cientificas, técnicas y financieras de que dispone la humanidad contemporánea para dar solución a aquellos problemas. Un ejemplo de estos esfuerzos es la serie de reuniones que vienen realizando el Consejo Mundial de la Iglesia y la Iglesia Católica, representada por la Comisión Pontificia Justicia y $\mathrm{Paz}^{11}$.

La división del mundo por razones ideológicas, no responde ya ni a los temas ni a los problemas contemporáneos. Pensadores $y$ hombres de acción de todos los rincones de la tierra - y cada vez en mayor cantidad - se hacen eco de la prédica incesante de las Naciones Unidas y' sus organismos especializados y se disponen a buscar formas de convivencia y colaboración que permitan canalizar esta cantidad jamás alcanzada de recursos financieros $y$ técnicos para el beneficio de la humanidad entera $y$ principalmente para los pueblos que aún no alcanzan los niveles minimos de desarrollo económico, social, culturál y politico ${ }^{12}$.

Se avisora como el surgimiento de un pensamiento humanista y social de alcance universal, de un hondo contenido pragmatico, que busca trascender las definiciones puramente conceptuales $y$ las buenas intenciones para realizar esfuerzos sinceros destinados a terminar con la miseria existente en todos los confines del planeta. Estos pensadores y hombres de acción se encuentran en América Latina, en Europa, en los Estados Unidos y en la Unión Soviética, se encuentran tanto en el mundo libre como en el mundo socialista, se encuentran en el mundo industrializado y en el mundo en desarrollo. Impresiona encontrar en todas partes del mundo una gran cantidad de dirigentes - hombres $y$ mujeres - entre $30 y^{\prime} 4^{0}$ años que están como preparados para entrar en acción en la nueva era que vive la humanidad.

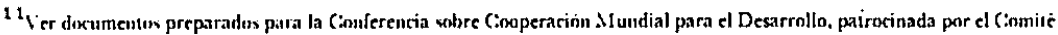
whre ueciedad. Desarrollo 、 Paz de la Comision Pantifucia Justicia y Paz v el Consejo . Hiundial té las Iglesias celehrada en

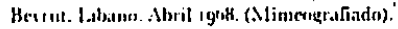

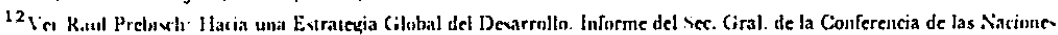

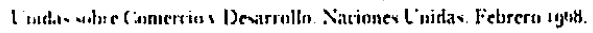




\section{La escala mundial.}

El mundo se ha unificado; se acortaron las distancias; se abrieron las comunicaciones; se acrecentaron los recursos técnicos y financieros. Si cada época de la historia de la humanidad tiene su propia escala, nuestra época ha llegado a la escala mundial. El mundo es la unidad base hasta para definir las politicas nacionales en perspectiva realista. Por eso, en este proceso de alcance mundial, es necesario que los dirigentes, en primer lugar, y los individuos en general, tomen conciencia de este nuevo hecho y se decidan a organizar las diversas sociedades de que forma parte teniendo bien presente esta nueva situación. En este mundo empequeñecido, interdependiente, unificado por las comunicaciones y que se mueve a velocidades supersónicas, los factores llamados internacionales adquieren una importancia excepcional y afectan de modo directo e inmediato aspectos claves de la convivencia universal: a) la paz y el desarme mundial con los esfuerzos consiguientes para establecer un sistema juridico eficiente destinado a controlar el desarme y' a neutralizar las áreas estratégicas polares y el espacio; b) la exploración y la conquista del espacio; c) el comercio mundial que, a través de los esfuerzos de la conferencia de las Naciones Unidas para el Comercio y Desarrollo, se trata de regular, asegurando beneficios justos para los paises en vias de desarrollo; d) el desarrollo de la ciencia y de la tecnologia; e) revolución en la información; f) las comunicaciones; g) la salud; h) la alimentación; i) la educación; j) la justicia mundial para el cumplimiento efectivo de la Declaración Universal de los Derechos Humanos, etc.

Es en estas áreas criticas para el futuro de la humanidad en las que se detectan las tendencias más marcadas hacia la constitución de organismos con autoridad mundial.

La reforma sustancial a la Carta de las Naciones Unidas es un hecho ya planteado ${ }^{13}$ y - sin duda - tomará en consideración la necesidad de legislar con alcance mundial algunas, si no todas, las áreas mencionadas precedentemente.

Se dijo anteriormente que cada época histórica tiene su propia escala. En el orden nacional, se comprenden muy bien las diferencias que existen entre lo que podria llamarse la escala municipal, la escala provincial y la escala nacional. Es obvio que no resulta lo mismo elaborar un plan para resolver problemas municipales o provinciales que elaborar planes para resolver problemas en escala nacional. Pero es también evidente que la dimensión nacional y $a_{a}$ no basta para planificar la economia ni la politica en su verdadero alcance por la dimensiọn que han adquirido los fenómenos

\footnotetext{
${ }^{13}$ Ver Clark, Grenville and Sohn, Louis: World Peace Through World Law. Ver además Norman Cousins, presidente de la Asociación Mundial de Federalistas Mundiales: Hav que fortalecer las Naciones Unidas. Discurso pronunciado en el Congreso Mundial de Oslo, 19237. Versión española de Arturo Orzabal Quintana. Departamento de Instrucción Pública. Puerto Rico. Igis.
} 
financieros, comerciales, industriales, técnicos, militares, económicos y politicos contemporáneos.

Los paises que pretendan modernizarse y prosperar y no presten la atención debida a las nuevas escalas regionales y mundiales, ya en vigencia, quedarán irremediablemente relegados a concepciones estrechas y sin viabilidad en el escenario mundial contemporáneo. Todo esfuerzo de planificación nacional de desarrollo deberá tener en cuenta las caracteristicas, los factores de poder y las tendencias del orden mundial que estamos viviendo. Si asi no se hiciere podrá caerse en una posición aislacionista absurda e innecesaria imponiendo graves cargas a los pueblos, o se conducirá a nuestros paises como a ciegas y sin rumbo dentro de la complejidad de este acelerado proceso de universalización mundial.

\section{La participación, signo del fuluro inmediato}

Por otra parte, pareceria que el signo de nuestro tiempo y de los años inmediatos por venir, será el signo de la participación. Los paises subdesarrollados lucharán para obtener mayor participación en las decisiones que afectan el comercio internacional; los paises sin armas nucleares procurarán tener mayor participación para asegurar el control.de la utilización de estas nuevas armas; los paises carentes de oro procurarán obtener condiciones de seguridad financiera frente a los que detentan el poder del oro; las minorias raciales de los Estados Unidos, Sud Africa y otrós paises del mundo continuarán su lucha por lograr el reconocimiento de un status de justicia; los trabajadores de todo el mundo insistirán en sus esfuerzos por participar más activamente en las responsabilidades de sus empresas, como asi también en el desarrollo de sus paises; los empresarios de todo el mundo procurarán participar en las decisiones que sobre la politica económica, bien sea de alcance nacional o bien regional y mundial, adopten sus gobiernos $y$ finalmente, las juventudes del mundo entero agotarán sus-esfuerzos por hacer oir su voz de esperanza y para participar en la creación del orden del futuro.

\section{LA INTEGRACIÓN DE AMÉRICA LATINA}

\section{El proceso de intégración latinoamericana dentro del proceso mundial de poder}

Es dentro de este proceso acelerado de universalización y de cambio donde se ubica el proceso de integración de América Latina y con respecto del cual tendrán que definir su participación los paises de América Latina. El proceso de integración de América Latina puede ser una respuesta al desafio de los tiempos que vivimos. Es una respuesta llena de riesgos $y$ de dificultades que hay que conocer y que hay que superar. Los riesgos provienen del hecho que las tendencias universalistas son muy fuertes $y$ pueden alterar la fisonomia $y$ el espiritu propio de los paises en vias de desarrollo transformándolos en 
meros números o cantidades de consumo negandoles toda posibilidad de expresión de sus valores culturales ! espirituales. Las dificultades surgen porque estos procesos despiertan resistencias en los sectores con intereses creados, que se escudan con las soberanias nacionales! para mantener el status quo. Pero, a pesar de los riesgos y las dificultades, es evidente que la integración de América Latina no agota sus temas fundamentales con la formación de un Mercado Común Latinoamericano que resuelva los problemas económicos más importantes. El proceso de unificación mundial que acabamos de mencionar puede afectar los valores básicos de la cultura latinoamericana e introducir corrientes de modernización ajenos - sino contrarios - a nuestro estilo de vida. De ahi que el movimiento de integración surja con una aparente contradicción que consiste en reconocer, por una parte, la importancia y el alcance irreversible de este proceso de unificación mundial $y$ por otra parte, la necesidad de salvar y proteger los valores fundamentales de nuestra cultura latinoamericana.

Por ello es que es necesario fortalecer el concepto de la integración liberadora por oposición al concepto de la inlegracion dependients, colonizadora o satelizante. Mientras los paises latinoamericanos no definan sus politicas fundamentales, tanto hacia dentro como hacia afuera, teniendo en cuenta la coỵuntura histórica en que vive el mundo moderno, no tendráı ubicación en los centros decisivos de poder con alcance mundial, no podrán participar con eficacia' en las discusiones ! en las decisiones que establecerán la estructura y condiciones generales del orden mundial del futuro.

Hay una correlación estrecha entre la toma de conciencia ! el conocimiento objetivo $y$ sistemático de la situación mundial - factores de poder; orgánización formal del poder; ! estructuración real del poder- - " una adecuada, evaluación que estos factores internacionales tienen sobre la vida de los paises, por una parte, ! la posibilidad de definir con acierto -realismo ! eficacia las politicas nacionales tanto de orden interno como de alcance mundial.

\section{Los organismos internacionales.}

Si se analiza la organización formal del poder en el mundo, vemos que nuestros paises forman parte de gran cantidad de organismos internacionales, de tipo politico, financiero, económico, comercial, militar, técnico, cientifico y cultural. Para dar sólo un ejemplo, Argentina forma parte de más de bo organismos internacionales y Chile de cerca de go. Pero ser miembro de estos orgañismos no es lo mismo que participar activamente en la elaboración de las politicas y en la dirección de los mismos. A veces se oyen criticas en contra de estos organismos internacionales o de sus politicas, como si no fueran nuestros propios gobiernos los que han contribuido a su creación; aportan recursos para su financiamiento y al mismo tiempo, frecuentemente, los que se niegan a participar eficientemente en ellos para 
que estos organismos cumplan la finalidad para la cual fueron creados. Es responsabilidad de los estados miembros que han aceptado formar parte de los organismos internacionales, participar activamente, con eficacia, en la definicion de las politicas de estos organismos ! controlar su funcionamiento. Para ello es necesario que nuestros paises definan sus politicas en escala mundial r que, logren una coordinación minima en la acción de sus embajadores $!$ representantes acreditados ante dichos organismos para que sus decisiones tengan un peso efectivo. En la esfera de la estructuración real dei poder mundial, respecto de la cual los organismos internacionales son sólo un aspecto, las decisiones de los gobiernos de América Latina tienen que canalizarse por otras vias, pero siempre suponen consenso ! coordinación de sus politicas, acción conjunta de los embajadores acreditados ante los diversos paises y coordinación con los organismos internacionales.

La fuerza del proceso de universalización es muy grande $y$ los paises de todas las regiones del mundo tendrán que definir sus politicas en función de sus propias posibilidades, distinguiendo muy bien los diversos planos en que la acción de estos paises puede ser conducida por ellos y aquellos planos de la acción que ya están dados por circunstancias ajenas a su control.

\section{El Mercado Común Latinoamericano}

El Mercado Común Latinoamericano, tal como lo definieron los presidentes de América Latina en la Reunión de Jefes de Estados Americanos, en Punta. del Este, en abril de 1967 , puede ser el instrumento que, debidamente constituido $y$ controlado por los paises latinoamericanos, amplie las bases de acción económica en el continente en beneficio de sus propios pueblos y fortalezca las bases para la programación de una acción politica latinoamericana en escala mundial.

\section{La Dimensión Continental}

La dimensıón continental es la que generalmente se considera como el ámbito propio de la integración de América Latiria, notándose una tendencia a concentrar la atención en esta dimensión continental, relegando en un segundo plano tanto los aspectos mundiales como los aspectos nacionales que condicionan el proceso de integración ${ }^{14}$

Un breve diagnóstico del proceso de integración de América Latina nos presenta los siguientes hechos principales:

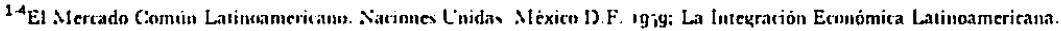
Haneo Nacional de Comercio Exterinl. S. A. Mexitr. Ig'; Felipe Herrera: America Latina inueyrada. Losada s.A. Buenns Aires, ighy: Intezracioun de Amerita Latina: experiencias r perspectivas. Fediciun preparada por Mliquel E. Wvontzek. C:ultura!

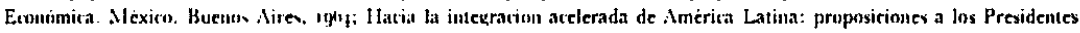

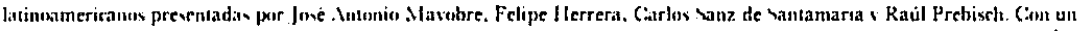

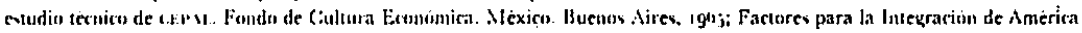

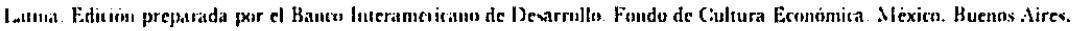
id'm.
} 
a) La situación de la ALALC y la necesidad de decidir sobre las condiciones de funcionamiento futuro de esta institución, incluyendo las posibilidades de vinculación más estrecha con el Mercado Común Centroamericano;

b) La acción del Banco Interamericano de Desarrollo en el campo de la integración $y$ su creciente acción financiera y promotora de la integración económica de América Latina;

c) Las gestiones que la nueva administración de la oEA realiza para asegurar el cumplimiento de la Declaración que los Presidentes de las Repúblicas de América Latina y de los Estados Unidos, firmaron en Punta del.Este en abril de igb7;

d) La constitución del llamado Grupo Andino como proyecto de integración subregional latinoamericano y que comprende Venezuela, Colombia, Ecuador, Bolivia y Chile;

e) La organización de la Cuenca del Plata, proyecto de dimensiones colosales que comprende Bolivia, Paraguay, Uruguay, Argentina y Brasil;

f) Proyectos más específicos como el de la carretera marginal de la selva del Perú y la formación de un grupo argentino, boliviano, paraguayo, uruguayo y peruano para construir un sistema de carreteras que sirvan de base a la llamada integración fisica del continente;

g) La creación de la flota de transporte aéreo latinnamericano y la de la nota de mar;

h) La creación de un sistema moderno de telecomunicación.

Estas son las instituciones y algunos proyectos que demuestran en formd categórica el dinamismo del proceso de integración de América Latina, como asi también las diversas áreas de actividad respecto de las cuales los paises - tanto en sus sectores gubernamentales como en sus sectores privadosnecesitan tener ideas claras que permitan una definición de sus politicas en forma coherente $y$ constructiva. Pero estas poltticas deben tener en cuenta la posición de América Latina en el sistema estratificado internacional, para que el esfuerzo conjunto pueda dar una respuesta al proceso atómico iufrido por la región.1.5

\section{II1. LA DIMENSIÓN NACIONAL DE LA}

INTEGRACIÓN LATINOAMERIGANA

\section{Integración para el desarrollo y la independencia.}

El primer problema que plantea la integración de América Latina con respecto a los intereses nacionales es el de su relación con el desarrollo $y$ la independencia:

La integracion económica de América Latina tiene que concebirse como un medio, un instrumento de los paises latinoamericanos para lograr las metas del desarrollo nacional. Se trata de un esfuerzo conjunto, para

\footnotetext{
${ }^{13} \mathrm{Ver}$ Censtavo Layns: La integración de América Latina v su inhuencia en el sistema internacinnal. en la Inegracioun de Amerirat.
} 
fortalecer $y$ acelerar los procesos de desarrollo nacionales y mejorar al todo mediante el progreso de cada una de las partes.

Esta coordinación de politicas, ! la adopción de politicas comunes deben ser decididas por los propios estados, y en uso de su propia soberania. No pueden existir esquemas impuestos desde afuera sin consultar los legitimos intereses nacionales. Pero por otra parte, los paises individualmente considerados, deberán tener en cuenta —en función del diagnóstico del poder mundial- cuáles son sus posibilidades reales y cuáles sus aspiraciones realizables $y$, sobre todo, cuál es la medida de su independencia real en temas politicos, económicos y militares. Porque la integración de América Latina deberá orientarse a asegurar la independencia en un mundo de paises independientes o la menor interdependencia en un mundo de creciente interdependencia ${ }^{1,6}$. Para lograr este objetivo —una vez más- hay que tomar conciencia de la ubicación que tiene cada pais dentro del sistema internacional estratificado. $Y$ es en función de las posibilidades reales de cada pais latinoamericano que deberá medirse la necesidad y la conveniencia de acciones conjuntas, concertadas en base a un programa de acción común, como la Comunidad Económica Latinoamericana.

\section{Algunas criticas a la integración de América Lalina.}

El estudio de las actitudes frente al proceso de integración de América Latina -tanto favorables como negativas $y$ correspondientes a gobiernoso instituciones privadas - ha sido analizada en otros trabajos $\stackrel{17}{\longrightarrow}$. Ahora interesa analizar algunas de las criticas que se han formulado en contra de la formación de un Mercado Común Latinoamericano, con el fin de examinar sus fundamentos y sacar de ellas todos los elementos positivos que contengan.

En primer lugar, la oposición más violenta a la integración de América Latina surge del sector comunista y los grupos afines de lá extrema izquierda revolucionaria, porque consideran que la formación de un Mercado Común Latinoamericano será "la última etapa en el proceso de corrupción total de la región impuesta por el imperialismo yanqui". En consecuencia; oponerse a la integración de América Latina, en el estado actual de los paises latinoamericanos, significaria oponerse al fortalecimiento de las clases dominantes, mientras se dan en el continente las circunstancias para unificarlo bajo dictaduras populares de liberación, apoỵadas por Moscú. La extrema izquierda revolucionaria habla de la unidad de América Latina bajo regimenes socialistas tipo Fidel Castro, a los que se llegará principalmente a través de la revolución violenta.

\footnotetext{
${ }^{16} \mathrm{Ver}$ Gustavo Lagos: International Stratification and Underdeveloped Countries. The Universitv or North Ca rolina Press. Igiz. Especialmente el capilulo It, punto 2 dedicado al estudio de la situación de dependencia de los paiscrsubdesarrollados: Wer también el interesante articulo de Marcos Kaplan: Estado, dependencia externa y desarrollo en América Latina en el $\mathrm{N}^{\circ} 2$, julioseptiembre ighol, pp. $179 / 213$.

${ }^{17}$ Ver nuestro trabajo: Actitudes frente a la Integración en el libro sobre La Integración Latinoamericana, situaciön $v$

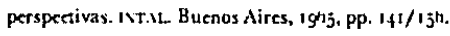


En Argentina se expresa otra corriente de opinión en una linea de pensamiento diametralmente opuesta a la anterior. Algunos sectores conservadores manifiestan su oposición a la integración de América Latina, porque consideran que Argentina es la continuación de Europa, que toda su grandeza la debe a su propio esfuerzo y que, en consecuencia, conviene a Argentina seguir la suerte del Viejo Continente, negociar todo lo posible en base bilateral con los Estados Unidos y dar la espailda a América Latina, con excepción de los paises limitrofes. Para esta posiciọn, la participación de la Argentina en el proceso de integración de América Latina significaria. incorporar muchas veces una carga muy pesada que retardaria el progreso de nuestro pais.

Por último, la posición de los sectores considerados como "industrialistas o desarrollistas" que se encuentran principalmente en Méjico, Brasily" 'Argentina y que parten de un acto de fe sobre las posibilidades futuras de sus respectivos paises, declarando que pueden obtenerse las metas de desarrollo nacional mediante un proceso de industrialización firme, que podria financiarse por medio del ahorro interno y recursos financieros externos en base a acuerdos bilaterales o internacionales. Este sector plantea en términos contradictorios el desarrollo nacional y la integración regional. Esta linea de pensamiento tiene vigencia en este momento $y$ es la inspiradora de las actitudes "frias" de los tres paises mencionados, con respecto a las reformas de ALALC $y$ otros temas de actualidad relacionados con la integración.

Creo que es esencial prestar seria atención a toda voz que se levante previniéndonos acerca de los riesgos, las diffcultades $y$ las consecuencias que un proceso de integración continental puede tener para los legitimos intereses nacionales. Considero acertada la opinión de los sectores comunistas y de la extrema izquierda latinoamericana cuando previenen contra alguna concepción de Mercado Común que estuviera inspirada solamente por los intereses de grandes firmas productoras extranjeras o, como ellos dicen, "por los intereses del imperialismo norteamericano". Es claro que, si asi fuera, se pondria en un riesgo inminente no sólo al desarrollo económico nacional sino que aumentaria la situación de dependencia de los paises y de la región en general. En consecuencia, es necesario esçuchar estas voces criticas para asegurar que la integración de América Latina será para servir los altos intereses latinoamericanos, y será realizada por latinoamericaños, sin perjuicio de la asistencia financiera que pudiera necesitarse.

La segurnda opinión critica, como dijimos, es tipicamente Argentina aunque también se encuentra entre algunos sectores del Uruguay.

Nadie puede negar la vinculación que la Argentina tiene con Europa, ni es necesario tampoco negar su carácter de pais fuertemente europeo pára que Argentina pueda participar en el proceso de integración. La vinculación de la Argentina con Europa, desconocida por unos y malentendida por otros, es no sólo cultural y económica, sino también étnica. Los argentinos son en su gran 
maỵoria exponentes directos de las razas que formaron Europa. Pero estos factores culturales y étnicos parecen no tener la fuerza suficiente como para inspirar a la Comunidad Económica Europea a que otorgue a la Argentina el trato que tan profundos lazos podrian justificar ${ }^{\mathbf{1}}$.

La Comunidad Económica Europea tiene fija sú atención en sus ex colonias del Africa y el Mercado Común Europeo fue concebido teniendo en cuenta la posibilidad de que participaran los paises africanos con un status éspecial. De modo que no basta que los argentinos "se sientan" europeos para que los europeos los tomen como tal. Esta actitud señala la incapacidad de estos sectores conservadores para identificarse con los paises latinoamericanos, hermanados por la raza, la cultura, el idioma, la religión, la historia y la geografia.

Por último, la posición industrialista y desarrollista es tal vez la más. articulada y la más moderna de estas posiciones criticas respecto a la integración y muchas de sus conclusiones son acertadas y hay que tenerlas bien presentes cuando se discutan las bases para la constitución del Mercado Común Latinoamericano o cualquiera otra definición de politica referente a este tema. Esta posición se ha defiriido contra lo que ha llamado "ciertos eșquemas de integración regional" caracterizados con las siguientes notas: primero, estarian inspirados en la división internacional del trabajo; segundo, deberian contar con órganos de gobierno dotados con poderes supranacionales que limitarian la soberania estatal, y tercero, serian incompatibles con el desarrollo nacional. La posición "desarrollista" se ha opuesto y se opone con muy buenos argumentos al esquema que acabamos de formular pero admite que la situación del mundo abre un amplio campo a la cooperación entre nuestras repúblicas y que es conveniente y necesario concentrar arreglos que conduzcan a la vigorización ! universalización del sistema de ALALC en toda América Latina, aceptando también la ejecución de los llamados proyectos multinacionales.

En otras palabras, acepta todo proyecto de integración y todo acto regional que fortalezca y acelere el desarrollo nacional, y rechaza todo proyecto de integración y todo acto regional que lo niegue o detenga el desarrollo nacional ${ }^{19}$. Asi formulado, se demuestra que el llamado sector desarrollista o industrialista se opone a un esquema de integración que no responde a los objetivós latinoamericanos. Pero una cosa es oponerse a un "esquema erróneo -exageradamente erróneo-de la integración y otra es oponerse al proceso de integración latinoamericana porque se plantea en términos contradictorios la relación integración regional y desarrollo nacional.

\footnotetext{
18Ver nuestro trabajo: La Iglesia y la integración de América Latina. Conferencia pronunciada en la Reunión Extrabrdinaria del Consejo Episcopal Latinoamericano. celay. Mar del Plata, Igith, publicado en Anuario de Sociologja de los Pueblos Ibéricos. Vol. ut. Instituto de Estudios Sindicales, Sociales y Cooperativi Iladrid, 19b7, Pp. $160 / 180$.

$19 \mathrm{Ver}$ Arturo Frondizi: El problema nacional de América Latina $y$ ciertos esquemas de integración regional en Estudios Internaoionales. Revista del Instituto de Estudios Internacionales de la Universidad de Chile. Ario $1 . N^{\circ}$ s. 3*4. Ortubre tg(37. Nasu igist. pp. $285 / 303$.
} 
La posición desarrollista tal como se expone en el articulo citado, se funda en un error de base que es considerar que el proceso de integración de América Latina responde a un modelo especifico, cerrado, preelaborado, $y$ con respecto al cual, los paises latinoamericanos no tienen otra alternativa que aceptarlo sumisamente y que ese modelo tendria las caracteristicas mencionadas. Puede afirmarse, sin embargo, que el proceso de integración de América Latina es un proceso abierto que está en plena elaboración y que no responde a modelos predeterminados.

Es precisamente, para evitar que la integración de América Latina se oriente en base a una división internacional de trabajo decretada por las grandes potencias; para apoyar la constitución de los mecanismos necesarios para que el Mercado Común - siendo común a todos los paises latinoamericanos- asegure la independencia de sus paises miembros y la de todo el continente, $y$ para que la integración de América Latina sirva al desarrollo nacional y continental y no se le oponga o lo limite, que se hace necesario aclarar honestamente las ideas, definir las posiciones en función de la situación mundial contemporánea y participar activamente en la construcción de la gran comunidad latinoamericana, de acuerdo a los objetivos definidos por los paises latinoamericanos.

\section{La Integración: proceso irreversible y tarea generacional}

El proceso de integración que está viviendo América Latina es irreversible. Podremos oir - de tiempo en tiempo- manifestaciones de algunos gobiernos contrarias a la continuación de este proceso. Pero para detener la marcha de la integración, los gobiernos que se oponen a ella tendrian que hacer tales esfuerzos conjuntos, que terminarian paradógicamente fortaleciendo los impulsos integracionistas.

La generación integracionistá está en la hora de la historia contemporánea y no habrá fuerza capaz de detenerla. Lo que si podria suceder, es que si algunos gobiernos insisten en oponerse a que una generación latinoamericana realice la integración para bien de los pueblos de América Latina, de pronto esos gobiernos se encuentran "integrados" por exigencias externas y bajo un orden mundial establecido por las superpotencias. En ese caso, la oposición ciega a la integración habria causado un daño irreparable a los pueblos de América Latina.

Pero felizmente los Presidentes de las Repúblicas de América Latina, reunidos en Punta del Este del 12 al 14 de abril de 1967 , decidieron solemnemente: "crear en forma progresiva, a partir de 1970, el Mercado Común Latinoamericano, que deberá estar sustancialmente en funcionamiento en un plazo no mayor de quince años". Y expresaron que "Esta magna tarea reforzará nuestros vinculos históricos, promoverá el desarrollo industrial y el fortalecimiento de las empresas industriales latinoamericanas, asi como una más eficiente producción y nuevas 
oportunidades de empleo y permitirá que la región desempeñe, en ẹ orden internacional, el papel destacado que le corresponde. Estrechará, en fin, la amistad de los pueblos del Continente"

Además, se comprometieron a construir las bases materiales de la integración económiea latinoamericana mediante la ejecución de proyectos multinacionales en materia de transportes, telecomunicaciones, energia, desarrollo de cuencas hidrográficas internacionales y regiones fronterizas; decidieron aunar esfuerzos para acrecentar los ingresos provenientes del comercio exterior; aumentar la productividad agropecuaria y la producción de alimentos; impulsar la educación; ampliar los programas nacionales sobre ciencia y tecnologia; incrementar los programas de mejoramiento de la salud y eliminar los pactos militares innecesarios.

Finalmente, los presidentes de las Repúblicas de América Latina expresaron su deseo de que el Tratado para la proscripción de Armas Nucleares en la América Latina entre en vigor a la brevedad posible, cumplidos los requisitos que el nuevo tratado establece.

Todos los temas de esta Declaración solemne, forman parte de un detallado Plan de Acción que los presidentes de las repúblicas latinoamericanas se han comprometido a realizar.

En ninguna parte de la Declaración ni en el Plan de Acción aprobados en Punta del Este, se habla de división internacional del trabajo ni de mecanismos supranacionales en los que los estados delegarian sus potestades soberanas. $Y$ este es el esquema el único esquema oficial de integración regional -aprobado por los gobernantes latinoamericanos. Este esquema tiene que ser cuidadosamente analizado $y$ si tiene errores, hay que denunciarlos y corregirlos. Pero no es correcto inventar esquemas de integración fundados en suposiciones o en opiniones erróneas, para someterlos a una critica fácil y luego fundarse en esa critica a un modelo falso o irreal, para criticar y oponerse al proceso de integración de América Latina, tal como viene dándose en la realidad.

Son cada dia más los intelectuales, profesionales, empresarios, dirigentes politicos $y$ sindicales, universitàrios y hombres de prensa que suman sus inquietudes y sus esfuerzos al proceso de integración de América Latina. Cada dia progresa más el análisis temático de la integración y son más numerosas las conferencias, las reuniones de trabajo y los seminarios que se celebran en diversas partes de América Latina dedicadas al estudio y al esclarecimiento de este proceso histórico.

La labor de la Secretaria Ejecutiva de la ALAlc y de la Secretaria del Mercado Común Centroamericano; las actividades del Banco Interamericano de Desarrollo relacionadas con el financiamiento de proyectos de integración $y$ las investigaciones y cursos que desarrolla a través del INTAL; los estudios de la CEPAL; la acción continua de varios organismos pertenecientes a todos los estados latinoamericanos; la creación del Comité 
de Acción para la Integración de América Latina y finalmente, y en una escala mucho más modesta, la contribución que la Escuela Latinoamericana de Ciencia Poltica y Administración Pública de Flacso, viene haciendo al proceso de integración mediante la preparación del primer anteproyecto concreto sobre constitución de la Comunidad Económica Latinoamericana, presentado al Parlamento Latinoamericano; todos estos y muchos otros más, son indicadores del volumen y de la intensidad que va adquiriendo el proceso de integración en América Latina.

Se ha dicho que para muchos paises de América Latina, la integración es todavia una quimera ${ }^{20}$. Usando el lenguaje de los estudios prospectivos, podemos decir que la integración de América Latina es una utopia accesible ${ }^{21}$. Su realización, con profundo sentido latinoamericano, dependerá de la vocación, del coraje y de la capacidad politica de esta generación latinoamericana.

\footnotetext{
${ }^{20}$ Stanley Hoftman, Obstinate or Obsolete? The Fate of the Nation State and the Case of Western Europe. Daedalus, 95, $\mathrm{N}^{\mathrm{N}}$ 3. Summer 1900.

${ }^{21}$ Ver Etapes de ta Prospective. Bibliotheque de Prospective. Presses Universitaires de France. 1907 , p. $34^{0}$.
} 